\title{
Bilateral Sudden Sensorineural Hearing Loss in Waldenström's Macroglobulinemia: Case Report and Review of the Literature
}

\author{
Nael M. Shoman ${ }^{1}$ Thamir Aldahmashi
}

Address for correspondence Nael M. Shoman, MD, FRCSC, Department of Otolaryngology-Head and Neck Surgery, Dalhousie University, 3184 Dickson Building, QEII Health Sciences Centre, 5820 University Avenue, Halifax, NS B3H 2Y9, Canada (e-mail: shoman@dal.ca).

\begin{abstract}
Keywords

- sudden sensorineural

- hearing loss

- Waldenström's

macroglobulinemia

Objective Waldenström's macroglobulinemia (WM) is a rare indolent B-cell lymphoproliferative disorder, representing 1 to $2 \%$ of all hematological malignancies. Involvement of the inner ear is rare with only case reports published over the past six decades. Methods The occurrence of bilateral sudden sensorineural hearing loss is exceeding rare, with limited published data in the literature. We present a case of a 62-year-old man diagnosed with WM who presented with bilateral sequential sudden sensorineural hearing loss.

Results A few months following his WM diagnosis, he experienced sudden hearing loss in his left ear. He was treated with a course of oral steroids with no improvement. Three months following this incident, he experienced a similar sudden loss of hearing in his right ear. Treatment for WM was initiated. A repeat hearing test, done 1 week later, did not show any significant improvement in his right hearing.

Conclusion The clinical course of WM is highly variable, with relatively infrequent involvement of lymph nodes, spleen, or liver. The inner ear is rarely involved. In this article, otologic clinical presentation is discussed, along with a review of the literature on hearing loss in WM.
\end{abstract}

\section{Introduction}

Waldenström's macroglobulinemia (WM) is an indolent B-cell lymphoproliferative disorder, characterized by a monoclonal immunoglobulin (Ig) M paraprotein. It was first described by Jan Gosta Waldenström in 1944 and classified as lymphoplasmacytic lymphoma (LPL) in the 2016 World Health Organization classification. ${ }^{1}$ It is a rare entity representing 1 to $2 \%$ of all hematological malignancies and $6 \%$ of the lymphoproliferative syndromes. ${ }^{2}$ The incidence is estimated to be three new cases per 1 million individuals, with men affected roughly twice as often as women, and the median age of diagnosis being 70 years. ${ }^{3}$ While the etiology remains unclear, various risk factors have been identified including the presence of monoclonal gammopathy of undetermined significance, family history, and autoimmune diseases., ${ }^{2,4}$

DOI https://doi.org/

10.1055/s-0040-1703970.

ISSN 2581-9607.
Clinical presentation is highly variable. Bone marrow infiltration can induce cytopenias; however, involvement of lymph nodes, spleen, or liver is less frequent than with other lymphomas. There have been reports of other organ involvement, including lungs, gastrointestinal tract, kidneys, skin, eyes, and the central nervous system. Clinical presentation as such can be attributable to tissue infiltration. In $15 \%$ of patients, hyperviscosity syndrome (HVS) is observed, caused by the monoclonal IgMs, resulting in increased red cell aggregation and decreased red cell deformability. ${ }^{5}$ Retinal hemorrhages or exudates may be seen, presenting as often-characteristic dilated and tortuous retinal veins. Neurological disease exists in approximately 16 to $20 \%$ of WM patients and can present with headache, ataxia, paresthesias, and dizziness. Involvement of the inner ear is relatively rare with only case reports published over the past six decades. ${ }^{6-8} \mathrm{~A}$ bilateral sudden 
sensorineural hearing loss (SNHL) in WM is even rarer, with very limited published data. ${ }^{9}$ We present a case of a patient with WM who presented with a bilateral sequential sudden SNHL, highlighting management and relevant literature.

\section{Case Report}

A 62-year-old man presented in late 2017 with spontaneous nosebleeds, fatigue, and peripheral lymphadenopathy. Blood work demonstrated elevated IgM levels, measuring $14.7 \mathrm{~g} / \mathrm{L}$, and he was subsequently diagnosed with IgM monoclocal gammopathy with positive MYD88 mutation, associated with lymphoplasmacytic lymphoma. His lymphoma was CD5 negative. A subsequent bone marrow biopsy showed positive involvement with lymphoma. Further blood work was unremarkable including negative anti-neutrophil cytoplasmic antibodies (ANCA), making vasculitis such as granulomatosis with polyangiitis a less likely cause for the epistaxis. His free kappa was normal, while free lambda was 63.2, with a kappa/ lambda ratio of 0.3 that is within normal limits. Total protein was 86 , albumin 38 , and uric acid 401 . Urinalysis demonstrated trace proteinuria and trace blood. The constellation of findings was most consistent with WM syndrome. Initial management was conservative with regular blood work. In January 2018, the patient had a syncopal episode and was admitted to hospital and diagnosed with hyperviscosity syndrome based on further elevation of his IgM level and was treated with one cycle of apheresis. He underwent an ophthalmologic assessment, and fundoscopy demonstrated tortuous retinal veins. Subjectively, he noted no acute or subacute visual changes. From an otologic standpoint, he has had no prior history of ear symptoms, ear infections, or ear surgery. He was aware of bilateral slowly progressive hearing loss over time, and his baseline audiometric testing showed bilateral normal hearing thresholds up to $2,000 \mathrm{~Hz}$, with a progressive decline in the higher frequencies to the mild range. Speech discrimination scores (SDS) were excellent, bilaterally. In December 2017, he experienced sudden hearing loss in his left ear, with no associated dizziness. A hearing test done at the time showed no change in his right hearing thresholds, while his left ear demonstrated mild low-frequency SNHL at $500 \mathrm{~Hz}$ with a precipitous drop to the moderately severe range, thereafter across all frequencies ( - Fig. 1). The SDS on the left dropped to $28 \%$. He was initiated on prednisone by his family physician, but he could only tolerate the prednisone for 4 days. The hearing did not improve, and he continued to cope with reliance on his right ear. In February 2018, he experienced a similar sudden loss of hearing in his right ear. He also experienced a mild sensation of disequilibrium and spatial disorientation with no true vertigo, and this was worse with positional head changes. He was referred for an urgent otolaryngology consultation. Initial otologic and neurotologic examination was unremarkable. The head thrust test showed a slight delay bilaterally. A repeat hearing test was done, which demonstrated right moderate high-frequency SNHL at $250 \mathrm{~Hz}$ with a precipitous drop to the severe range across the middle and high frequencies. The SDS dropped to $24 \%$. A magnetic resonance imaging (MRI) study of the brain was done, and this did not demonstrate any retrocochlear pathology or signs of cochlear hemorrhage. He was placed on a 7-day course of prednisone and referred back for urgent reevaluation by his hematologist. A repeat hearing test done 1 week later showed only significant improvement in his left hearing at $2,000 \mathrm{~Hz}$ (-Fig. 2). The patient went on to receive six cycles of bendamustine with rituximab, and did well with no significant side effects. A hearing test repeated every 3 months did not demonstrate a change in hearing up to a year later, and he continues to use bilateral hearing aids with benefit.

\section{Discussion}

The patient in this case presented with classic features of WM, with onset in the seventh decade, spontaneous epistaxis, fatigue, and lymphadenopathy. The presentation of sudden SNHL in patients with WM is rare, and the etiology is unclear. The first report published on a patient with WM and sudden bilateral SNHL by Afifi and Tawfeek proposed a hemorrhagic etiology, ${ }^{10}$ based on the observation of an abnormal

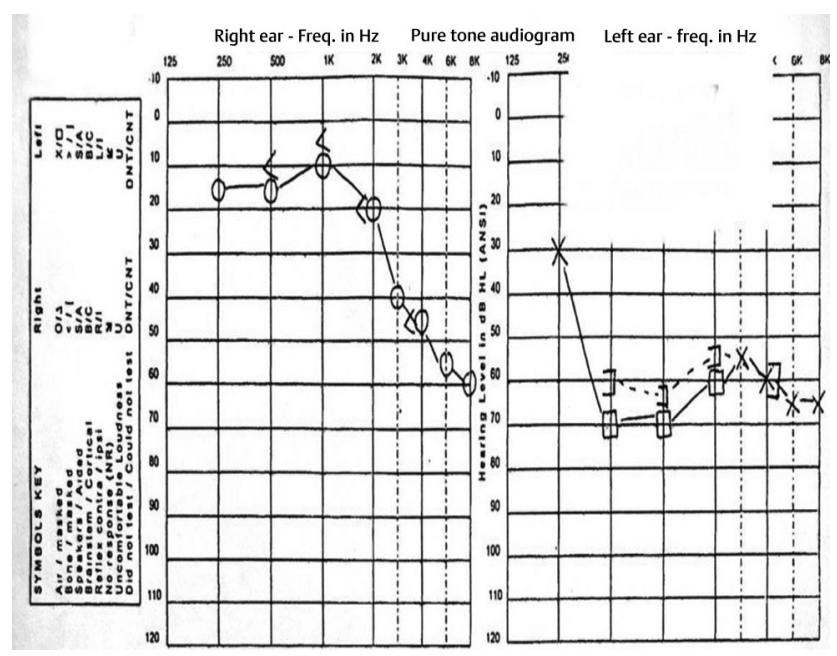

Fig. 1 Baseline audiogram when the patient was referred to otology clinic. CNT, could not test; DNT, did not test.

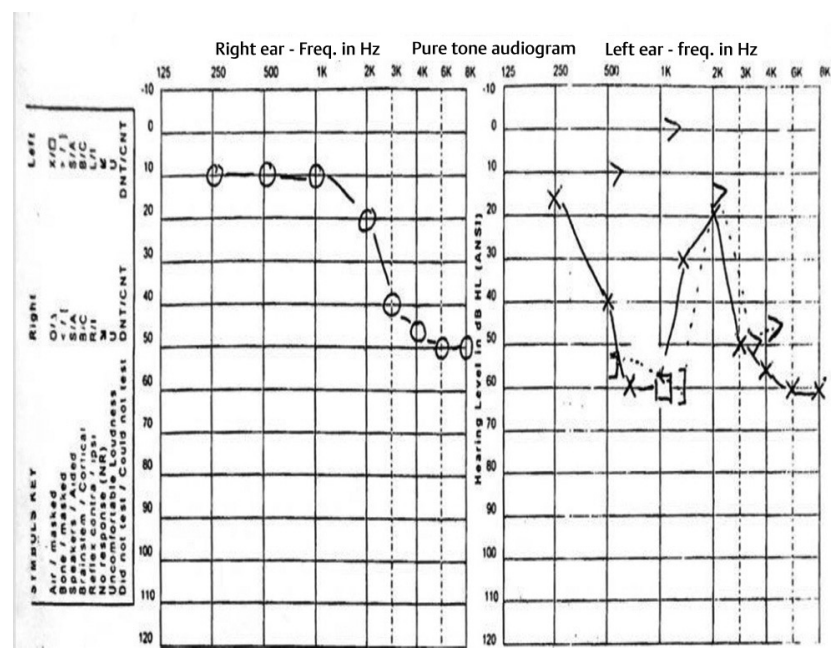

Fig. 2 Audiogram after the patient received a 7-day prednisone course showing no significant improvement. CNT, could not test; DNT, did not test. 
coagulation profile with prolonged bleeding time. This was also illustrated in a temporal bone biopsy in one of the cases by Wells et al. ${ }^{11}$ Subsequent reports of the hearing loss in WM have attributed blood hyperviscosity and clot formation as a more likely etiology. Factors influencing blood viscosity include hematocrit value; fibrinogen levels; red blood cell filterability, which partially reflects their deformability; red cell aggregation; plasma globulin levels; and platelet aggregation. ${ }^{12}$ As the blood viscosity increases, its velocity may decrease in the microcirculation, and that raises the potential of local sludging, microthrombi development, and epithelial damage. ${ }^{7}$ This plausible mechanism then makes the hearing loss potentially reversible. In four cases of sudden SNHL presented by Wells et al, three demonstrated a reversal of the hearing loss, and the authors proposed hyperviscosity and blood stagnation in the cochlear venous system as a mechanism. ${ }^{11}$ Ruben et al presented the case of a patient with bilateral sequential sudden SNHL, and proposed a similar mechanism for the hearing loss in WM. ${ }^{6}$ Similarly, in a case presented by Platia and Saral, the lack of progression of the hearing loss in a patient with WM treated with longterm anticoagulation supported the hypothesis that the mechanism entails cochlear venous thrombosis. ${ }^{8}$ Syms et al presented the case of a 72-year-old woman who presented with subacute onset of imbalance and bilateral diminution of hearing. ${ }^{7}$ She was treated with seven cycles of fludarabine, with significant improvement of hearing across all frequencies and in her SDSs after return to normal viscosity. Based on the understanding then of the likely pathophysiology of hearing loss in the setting of WM, and the limited literature available on the subject, it would seem that steroids do not have a significant benefit. This was also the experience with the patient in the current case who did not have a significant improvement in hearing with a week course of oral prednisone despite being treated within a week of acute onset. Neurological symptoms in WM tend to improve with the management of the underlying condition. ${ }^{13}$ Review of published cases on acute and subacute SNHLs in WM also shows a tendency for improvement with the management of the underlying condition and improvement of the hyperviscosity. ${ }^{7}$ While some reports have found no hearing improvement despite aggressive treatment, ${ }^{9}$ it is notable that MRI scanning showed findings reflective of likely hemorrhage into the inner ear, which may not be as responsive then to treatment.

Because the cochlea is susceptible to ischemia, especially at the level of the organ of Corti, this mechanism may partly explain the preferential effect of WM-related ischemia on the cochlea, and particularly the higher frequencies. Indeed, review of previous case reports showed patients mostly presented with cochlear, as opposed to cochleovestibular symptoms, as was with the case presented in the current report. Through similar pathophysiology, the retina is also supplied by an end artery that demonstrates hyperviscosity-related retinopathy in WM syndrome patients, seen in prior case reports, as well as in the case reported here. ${ }^{14-16}$ Because the retina is readily accessible through fundoscopic examination, it could potentially serve as a means of screening for the deleterious effects of HVS by the demonstration of tortuous retinal veins of the retina, as was seen in this patient. Menke et al demonstrated that the initial manifestations of HVSrelated retinopathy in WM can be seen in the peripheral retina using indirect ophthalmoscopy in patients with serum viscosity levels as low as $2.1 \mathrm{cp}$ and IgM levels as low as 2,950 $\mathrm{mg} / \mathrm{dL} .{ }^{17}$ The location of these changes in the peripheral retina renders them asymptomatic and unnoticed by the patient early on, and so this can potentially help in the early detection of HVS, and initiation of treatment to prevent irreversible damage to hearing and vision. ${ }^{17}$ Likewise, treatment of WM and normalizing the SV in patients with acute hearing changes can result in considerable improvement in hearing. ${ }^{7}$ In our presented case, the hearing remained stable for the follow-up period of 1 year following treatment of WM. Based on the current literature review, the most effective treatment for WM-related hearing loss is to treat the underlying cause, which highlights the importance of close collaborative management involving the otolaryngologist and the hematologist. Patients newly diagnosed with WM should be counseled about the potential risks of visual and hearing changes so as to seek prompt evaluation in the event of subjective changes. Regular screening for retinal changes can help detect and potentially initiate treatment that could prevent progression of HVS and the risk of hearing loss.

\section{Conclusion}

While sudden SNHL is rare in WM, it is important for the consulting otologist to be aware of this association and its unique pathophysiology and management strategy. Counseling of patients newly diagnosed with WM regarding the potential risk of SNHL and advocacy for regular audiometric testing should be encouraged by the otologist as part of the multidisciplinary management of these patients.

\section{Funding \\ None. \\ Conflict of Interest \\ None declared.}

\section{References}

1 Swerdlow SH, Campo E, Pileri SA, et al. The 2016 revision of the World Health Organization classification of lymphoid neoplasms. Blood 2016;127(20):2375-2390

2 Simon L, Baron M, Leblond V. How we manage patients with Waldenström macroglobulinaemia. Br J Haematol 2018;181(6):737-751

3 Castillo JJ, Olszewski AJ, Cronin AM, Hunter ZR, Treon SP. Survival trends in Waldenström macroglobulinemia: an analysis of the Surveillance, Epidemiology and End Results database. Blood 2014;123(25):3999-4000

4 Treon SP, Hunter ZR, Aggarwal A, et al. Characterization of familial Waldenstrom's macroglobulinemia. Ann Oncol 2006;17(3):488-494

5 Kwaan HC, Bongu A. The hyperviscosity syndromes. Semin Thromb Hemost 1999;25(2):199-208

6 Ruben RJ, Distenfeld A, Berg P, Carr R. Sudden sequential deafness as the presenting symptom of macroglobulinemia. JAMA 1969;209(9):1364-1365 
7 Syms MJ, Arcila ME, Holtel MR. Waldenstrom's macroglobulinemia and sensorineural hearing loss. Am J Otolaryngol 2001;22(5):349-353

8 Platia EV, Saral R. Deafness and Waldenström's macroglobulinemia. South Med J 1979;72(11):1495-1496

9 Shibata DK, Johnston SC. Bilateral sudden hearing loss in Waldenstrom's Macroglobulinemia: MR appearance. Radiol Case Rep 2015;1(3):77-79

10 Afifi AM, Tawfeek S. Deafness due to Waldenstrom macroglobulinaemia. J Laryngol Otol 1971;85(3):275-280

11 Wells M, Michaels L, Wells DG. Otolaryngological disturbances in Waldenstrom's macroglobulinaemia. Clin Otolaryngol Allied Sci 1977;2(4):327-338

12 Hall SJ, McGuigan JA, Rocks MJ. Red blood cell deformability in sudden sensorineural deafness: another aetiology? Clin Otolaryngol Allied Sci 1991;16(1):3-7
13 Baehring JM, Hochberg EP, Raje N, Ulrickson M, Hochberg FH. Neurological manifestations of Waldenström macroglobulinemia. Nat Clin Pract Neurol 2008;4(10):547-556

14 Kim W, Kweon E. Images in Clinical Medicine. Hyperviscosity-related retinopathy in Waldenström's macroglobulinemia. N Engl J Med 2016;374(1):73

15 Kudo N, Usui M, Nakabo Y, et al. Waldenstrom's macroglobulinemia: a report of two cases, one with severe retinopathy and one with renal failure. Case Rep Hematol 2017;2017:3732902

16 Agorogiannis E, Sultan Z, Ramasamy B. Peripheral retinopathy associated with Waldenström's macroglobulinaemia. Postgrad Med J 2017;93(1104):641

17 Menke MN, Feke GT, McMeel JW, Branagan A, Hunter Z, Treon SP. Hyperviscosity-related retinopathy in waldenstrom macroglobulinemia. Arch Ophthalmol 2006;124(11):1601-1606 